\title{
Y la Reina preguntó: ¿Por qué los economistas no han sido capaces de predecir la crisis económica? ¿Será quizás que no leen la prensa? ... La importancia de usar las noticias de prensa en la docencia de Economía
}

\author{
María Rosalía Vicente Cuervo \\ Universidad de Oviedo \\ mrosalia@uniovi.es
}

Recibido: $11 / 10 / 2012$

Aceptado: 23/01/2013

\begin{abstract}
Resumen
Una de las principales críticas de la que han sido objeto tanto la profesión de economista como la formación que éstos reciben es su desconexión con los problemas del mundo real unida al predominio del formalismo, la sofisticación técnica y la complejidad matemática. Siguiendo las recomendaciones de algunos de los Premios Nobel en Economía (Coase, Friedman y Krugman, entre otros), que insisten en la importancia de que la docencia en Economía esté ligada a problemas reales, este trabajo pretender ilustrar cómo es posible vincular la enseñanza de materias técnicas como la Estadística y la Econometría con la realidad a través del uso de las noticias de prensa.

Palabras clave: docencia, noticias de presa, Economía, Estadística
And the Queen asked: Why economists haven't been able to predict the economic crisis? Don't they read the news? ... The importance of using news when teaching Economics

\begin{abstract}
One of the main criticisms made to the profession and training of economists is related to the fact that this discipline has been become detached from real-world problems, while it has focused on formalism, technical sophistication, and mathematical complexity. Following the suggestions of some Nobel Prizes in Economics (Coase, Friedman and Krugman, among others), who highlight the importance of linking teaching and real-world problems, the aim of this paper is to show some examples of how to teach quantitative methods in Economics by using news articles.
\end{abstract}

Keywords: teaching, news, Economics, Statistics

\section{Referencia normalizada}

VICENTE CUERVO, María Rosalía (2013): "Y la Reina preguntó: ¿Por qué los economistas no han sido capaces de predecir la crisis económica? ¿Será quizás que no leen la prensa? ... La importancia de usar las noticias de prensa en la docencia de Economía”. Estudios sobre el Mensaje Periodístico. Vol. 19, Núm. especial marzo, págs.: 523-530. Madrid, Servicio de Publicaciones de la Universidad Complutense.

Sumario: 1. Introducción. 2. Metodología. 3. Desarrollo; 1.3. Distinción censo/muestra: elecciones Obama-Romney; 3.2. Números índices y deflactación: los 10 años del euro; 3.3. Medidas de desigualdad y pobreza: ¿qué está pasando en España a raíz de la crisis?. 4. Conclusiones. 5. Referencias bibliográficas

\section{Introducción}

En el año 2009 en una visita que la reina de Inglaterra realizó a la prestigiosa London School of Economics (LSE), ésta preguntó el porqué de que tantos economistas no hu- 
biesen sido capaces de predecir la grave crisis económica que se estaba sufriendo a nivel mundial.

Su pregunta recibió dos cartas de respuesta: una primera de los profesores Tim Besley y Peter Hennessy, y una segunda de un grupo más amplio de economistas (Sheila C. Dow, Peter E. Earl, John Foster, Geoffrey C. Harcourt, Geoffrey M. Hodgson, J. Stanley Metcalfe, Paul Ormerod, Bridget Rosewell, Malcolm C. Sawyer, y Andrew Tylecote) que consideraban que la primera carta era demasiado autocomplaciente y carecía de una autocrítica básica tanto en cuanto al ejercicio de la profesión como a la formación que recibían los economistas, ambas alejadas de la realidad y centradas en alcanzar la perfección técnica y matemática (Dow et al., 2009). En palabras de estos economistas "esta formación estrecha (limitada) (...) que se centra en técnicas matemáticas y en la construcción de modelos formales empíricamente no controlados es uno de los motivos más importantes que explica el fracaso de nuestra profesión" (Dow et al., 2009: web).

Este alejamiento de la realidad ya venía advirtiéndose desde hacía más de una década. En 1991 la comisión creada por la Asociación Americana de Economía para el análisis de los estudios de grado en las facultades americanas, puso de manifiesto el riesgo de que "los programas de licenciatura podrían generar una generación con demasiados tontos sabios, hábiles en técnicas pero inocentes cuando se traten de asuntos económicos reales" (Dow et al., 2009: web). También las críticas de algunos Premios Nobel de Economía como Coase y Friedman apuntaban en esa misma dirección (Cuadrado Roura, 2010). En concreto, Friedman consideraba que "la economía se ha ido convirtiendo cada vez más en una rama arcana de las matemáticas en vez de ocuparse de problemas económicos reales" (Friedman, 1999: 137).

Para Paul Krugman, Premio Nobel de Economía en 2008, la solución a este problema es muy sencilla: leer, leer y tres veces leer la prensa con regularidad. "Mi director de tesis, Rudy Dornbusch, me dijo una vez: antes de escribir artículos académicos, no leas revistas especializadas, ni repases la última teoría. Pásate unos meses leyendo periódicos e historia porque la mejor guía para la investigación económica es el mundo real" (Krugman, 2012: web).

Parafraseando a Krugman, en este trabajo queremos mostrar cómo el mundo real no es sólo la mejor guía para la investigación económica sino también para la docencia en Economía, incluso para aquellas enseñanzas más técnicas dentro de la carrera como pueden ser la Estadística y la Econometría. Para ello en el siguiente apartado se exponen cuáles son los principales métodos docentes que se están aplicando en las facultades de Economía, para luego ofrecer algunos ejemplos concretos de cómo se puede vincular la enseñanza de las técnicas cuantitativas empleadas en el ámbito económico con noticias de actualidad de tal forma que para los alumnos sea más sencillo comprender la utilidad de estos métodos.

\section{Metodología}

Una de las quejas persistentes entre los alumnos de las facultades de Economía se refiere al excesivo contenido teórico de las enseñanzas universitarias y a la desconexión con el mundo profesional. Según un estudio de la Fundación BBVA (2010), el 
$62 \%$ de los estudiantes de Económicas en España considera que la universidad no les prepara adecuadamente para el ejercicio de su futura actividad profesional.

Si revisamos los métodos docentes que se emplean en las facultades de Economía nos encontramos, tal como ilustra la siguiente tabla, que hay un predominio de los sistemas tradicionales como la lección magistral, mientras que recursos más interactivos y que acerquen a los estudiantes a la realidad profesional (como pueden ser las simulaciones) son aún empleados de forma minoritaria entre los docentes. Los datos de la tabla reflejan la situación en las aulas universitarias de los Estados Unidos a falta de un trabajo similar para el caso español. Los datos corresponden a un trabajo realizado por los economistas norteamericanos Watts y Schaur (2011) que desde mediados de los años noventa vienen estudiando los métodos y recursos docentes empleados por el profesorado de las facultades de Economía de los Estados Unidos. Entre los materiales empleados vemos que los artículos de prensa se emplean de media en un 25 por ciento de las clases de los cursos introductorios e intermedios, para aumentar hasta el 34 por ciento en algunas materias específicas, y alcanzar un valor de sólo el 13 por ciento en las materias más técnicas, Estadística y Econometría.

Tabla 1: Métodos y recursos docentes en las facultades de Economía americanas. (\% de clases dedicadas durante el semestre) Mediana (media). Año 2010. Nota: A: Cursos introductorios (principios de microeconomía, macroeconomía...); B: Cursos intermedios de teoría; C: Estadística y Econometría; D: Materias específicas. Fuente: Adaptado de Watts y Schaur (2011).

\begin{tabular}{|c|c|c|c|c|}
\hline \multirow{3}{*}{\begin{tabular}{|l|} 
Métodos y recursos para las clases: \\
\end{tabular}} & \multicolumn{4}{|c|}{ Tipo de materia } \\
\hline & \multirow[t]{2}{*}{$\mathbf{A}$} & \multirow[t]{2}{*}{$\mathbf{B}$} & \multirow[t]{2}{*}{$\mathbf{C}$} & \multirow[t]{2}{*}{$\mathbf{D}$} \\
\hline & & & & \\
\hline Lección magistral & $83(65)$ & $83(65)$ & $83(63)$ & $83(62)$ \\
\hline Empleo de la pizarra & $83(57)$ & $83(60)$ & $83(59)$ & $67(54)$ \\
\hline Presentaciones animadas & $22(37)$ & $6(28)$ & $22(38)$ & $22(34)$ \\
\hline Transparencias & $0(15)$ & $0(12)$ & $0(12)$ & $0(13)$ \\
\hline DVDs, videos, películas & $6(9)$ & $0(3)$ & $0(2)$ & $6(7)$ \\
\hline \multicolumn{5}{|l|}{ Debate/discusión: } \\
\hline Entre alumnos & $6(18)$ & $6(14)$ & $6(15)$ & $14(23)$ \\
\hline Profesor-alumno & $50(43)$ & $50(42)$ & $50(43)$ & $50(49)$ \\
\hline Votaciones & $0(6)$ & $0(6)$ & $0(3)$ & $0(3)$ \\
\hline \multicolumn{5}{|l|}{ Otras actividades en clase: } \\
\hline Experimentos & $6(11)$ & $0(7)$ & $0(6)$ & $0(7)$ \\
\hline Juegos y simulaciones & $6(8)$ & $0(6)$ & $0(7)$ & $0(7)$ \\
\hline Aprendizaje cooperativos/trabajo en grupo & $6(16)$ & $6(15)$ & $6(17)$ & $6(21)$ \\
\hline Prácticas de laboratorio & $0(8)$ & $0(6)$ & $50(40)$ & $0(8)$ \\
\hline Test de autoevaluación para el estudiantes & $0(6)$ & $0(3)$ & $0(4)$ & $0(4)$ \\
\hline Referencias a cuestiones de género, raciales... & $6(12)$ & $0(7)$ & $6(10)$ & $6(15)$ \\
\hline Referencias a la literatura, teatro, música... & $6(10)$ & $6(7)$ & $0(4)$ & $6(9)$ \\
\hline
\end{tabular}




\begin{tabular}{|l|c|c|c|c|c|}
\hline Referencias al deporte & $6(11)$ & $6(9)$ & $6(11)$ & $6(10)$ \\
\hline Estudio de la vida de economistas relevantes & $6(9)$ & $6(8)$ & $0(7)$ & $6(11)$ \\
\hline Otros & $0(9)$ & $0(3)$ & $0(4)$ & $0(4)$ \\
\hline Materiales: & \multicolumn{5}{|l|}{} \\
\hline Manuales & $83(73)$ & $83(73)$ & $83(71)$ & $83(64)$ \\
\hline Libros de ejercicios & $6(19)$ & $0(16)$ & $0(12)$ & $0(7)$ \\
\hline Apuntes elaborados por el profesor & $22(36)$ & $22(40)$ & $22(41)$ & $22(40)$ \\
\hline Ejercicios elaborados por el profesor & $50(48)$ & $83(57)$ & $83(60)$ & $50(51)$ \\
\hline Artículos de prensa & $22(28)$ & $6(25)$ & $6(13)$ & $22(34)$ \\
\hline Artículos científicos & $0(6)$ & $6(10)$ & $6(21)$ & $22(37)$ \\
\hline Búsqueda de datos en: & \multicolumn{5}{|l}{} \\
\hline Biblioteca & $0(9)$ & $0(9)$ & $6(18)$ & $6(25)$ \\
\hline Internet & $6(19)$ & $6(16)$ & $22(30)$ & $22(32)$ \\
\hline CDs & $0(1)$ & $0(1)$ & $0(4)$ & $0(3)$ \\
\hline Búsqueda de bibliografía en: & & & & \\
\hline Biblioteca & $0(6)$ & $0(7)$ & $0(16)$ & $6(27)$ \\
\hline Internet & $0(9)$ & $0(9)$ & $0(19)$ & $22(28)$ \\
\hline EconLit & $0(4)$ & $0(6)$ & $0(18)$ & $6(23)$ \\
\hline
\end{tabular}

Si bien, a primera vista, puede parecer difícil la manera en que se pueden emplear las noticias de prensa para enseñar técnicas cuantitativas, algunos prestigiosos profesionales en estas áreas, como es el caso de los profesores William E. Becker y William H. Greene, llevan tiempo trabajando en esta dirección (Becker, 1997, 2002a, b; Becker y Greene, 2001; Becker y Watts, 2001). De igual forma, la Asociación Americana de Estadística (2010) insiste en la necesidad de que la enseñanza de estas técnicas siempre se plantee en el contexto de problemas reales.

En el siguiente apartado trataremos justamente de ilustrar cómo es posible vincular la enseñanza de las herramientas estadísticas a las noticias de la actualidad económica y social. En concreto, nos centraremos en los siguientes conceptos estadísticos: la diferencia entre los censos y las muestras, los números índices, el concepto de deflactación, y las medidas de desigualdad y pobreza.

\section{Desarrollo}

\subsection{Distinción censo/muestra: elecciones Obama-Romney}

En cualquier curso introductorio de Estadística una de las primeras distinciones básicas se refiere a la diferencia entre censo y muestra. Se habla de censo cuando se dispone de información de todos y cada uno de los elementos de la población objeto de estudio, mientras que en el caso de las muestras, únicamente se dispone de información de una parte representativa de la población. ¿Cómo podemos ilustrar estos conceptos y las ventajas e inconvenientes asociados a trabajar con censos y muestras a partir de la realidad? Quizás una manera muy sencilla consista en recurrir a las noti- 
cias actuales sobre las elecciones presidenciales de los Estados Unidos donde se presentan como candidatos: Obama, a la reelección, y Romney. Los sondeos electorales no son otra cosa más que una encuesta de carácter muestral; con una población de más de 200 millones de personas con derecho a voto en los Estados Unidos, es muy intuitivo para un alumno entender por qué no es posible que un sondeo se plantee como una encuesta de tipo censal por todos los costes que lleva asociados en forma de tiempo, dinero, recursos humanos... Además revisando la ficha técnica de alguno de los sondeos se puede aprovechar para explicar la fórmula de cálculo del tamaño de muestra, que no indica otra cosa más que el número mínimo de personas a encuestar, y también cómo ese tamaño puede modificarse a partir de cambios en el margen de error y el nivel de confianza.

\subsection{Números índices y deflactación: los 10 años del euro}

Un segundo grupo de conceptos fundamentales es el que se refiere a aquel conjunto de medidas que nos permiten estudiar la evolución de las magnitudes en el tiempo. El instrumento más sencillo es el número índice, un simple cociente entre los valores de una magnitud en dos momentos en el tiempo. Según el valor resultante sea mayor, menor o igual que uno, la magnitud habrá aumentado, disminuido o se habrá mantenido constante entre los dos periodos considerados. A pesar de lo intuitivo de los números índices a los estudiantes es un concepto que les suele resultar de gran dificultad. Quizás por ello sea vital explicarlo a partir de las noticias de actualidad, de temas que les lleguen a los alumnos y que puedan facilitar su comprensión. Con tal objetivo podríamos referirnos al estudio que elaboró la Organización de Consumidores y Usuarios (2011) con motivo de los diez años del euro y en el que se estudia cuál ha sido la evolución de los precios de consumo durante el periodo 2002-2011, destacando un incremento de los precios de alimentos como los huevos de casi un $115 \%$ (de 7 céntimos de euro por unidad han pasado a costar 15 céntimos de euro) (Organización de Consumidores y Usuarios, 2011).

Este mismo estudio puede además servirnos para introducir otro concepto esencial como es la deflactación. Dicho de una forma muy sencilla, deflactar consiste en eliminar el efecto de la inflación de una magnitud monetaria. Los estudiantes de Economía están familiarizados, si bien no tanto con este término de deflactación, con los relativos a magnitudes económicas como el Producto Interior Bruto valoradas a precios corrientes (o lo que es lo mismo, en términos nominales, que incluyen el efecto de la inflación) y magnitudes a precios constantes (o en términos reales de las que se ha descontado el efecto de la inflación). Sin embargo, por importante que sea el Producto Interior Bruto, es algo que les resulta abstracto, demasiado lejos de su realidad, por ello puede ser interesante ilustrar el efecto que tiene la inflación a lo largo del tiempo a partir de algo como la ganancia o pérdida de poder adquisitivo. El mencionado estudio de la Organización de Consumidores y Usuarios (2011) incluye un análisis comparativo de la evolución de los salarios de los españoles y la de los precios, llegando a la conclusión de que se ha sufrido una enorme pérdida de poder adquisitivo a lo largo de los diez últimos años. 


\subsection{Medidas de desigualdad y pobreza: ¿qué está pasando en España a raíz de la crisis?}

Un tercer grupo de conceptos básicos para los estudiantes de Economía son las medidas de desigualdad y pobreza, cuyos objetivos son cuantificar, respectivamente, el nivel de desigualdad existente en el reparto de una magnitud monetaria entre los componentes de una población (ya sea renta, ingresos, o Producto Interior Bruto, por ejemplo) y determinar el nivel de pobreza.

Uno de los principales instrumentos analíticos para medir la desigualdad es el índice de Gini, un índice que toma valores entre cero y uno, donde el cero representa una situación de equidistribución (reparto igualitario) y uno, la máxima desigualdad, caso en el que toda la riqueza/renta estaría en manos de un único individuo. Este índice suele ir acompañado de la representación gráfica de la curva de Lorenz, que muestra en términos porcentuales cómo se distribuye la riqueza entre una población que previamente ha sido ordenada en función de su renta de menor a mayor. De esta forma cada punto de la curva de Lorenz muestra el porcentaje de riqueza que acumulan los " $\mathrm{i}$ " primeros individuos menos ricos.

En cuanto a la pobreza, la forma básica para estudiarla es a partir de la determinación del llamado umbral de riesgo de pobreza, el valor de los ingresos por debajo de cual se considera que una familia o individuo se encuentra en situación de riesgo de pobreza. Este umbral se determina como un determinado porcentaje sobre el ingreso mediano, en concreto y siguiendo las recomendaciones de Eurostat "este umbral se fija en el 60\% de la mediana de los ingresos por unidad de consumo de las personas" (Instituto Nacional de Estadística, 2012: 2).

En la actual situación de crisis económica, los efectos que está teniendo sobre la desigualdad y la pobreza son temas recurrentes en las noticias no sólo de la prensa especializada, sino también de la prensa generalista, ya sea escrita o digital y en los telediarios (Martínez Fernández et al., 2012). Si bien los estudiantes tienen en su cabeza una idea general sobre lo que está ocurriendo en términos de desigualdad y pobreza (que está aumentando), distan mucho de conocer cómo se miden. Por ello, es interesante aprovechar la abundancia de noticias, para seleccionar algunos artículos y leyéndolos en clase introducir las técnicas e instrumentos para medir la desigualdad. También se pueden aprovechar los suplementos especiales de algunos diarios. Así, por ejemplo, El País (2011) tiene elaborados una serie de gráficos que ilustran la evolución de la desigualdad en España desde el año 2000 a partir del índice de Gini y de la ratio correspondiente al 20 por ciento de la población de mayores/menores ingresos, que es otra de las medidas habituales de la desigualdad. Estas mismas medidas se incluyen en el informe de la Fundación FOESSA (2012), que constituye uno de los principales referentes en el estudio de la exclusión social en España.

\section{Conclusiones}

La Economía como ciencia ha sufrido en los últimos años numerosas críticas centradas en el predominio del formalismo técnico frente a la profunda comprensión y análisis de los fenómenos económicos del mundo real. Prueba de ello es la actual crisis económica que muchos profesionales de la Economía no fueron capaces de ver. Son numerosas las voces críticas que se han levando y que exigen la necesidad de reo- 
rientar tanto la profesión como la formación de los economistas para acercarlos al mundo real. Centrándonos en el último aspecto, el de la formación, este artículo pretende ofrecer algunos ejemplos muy sencillos de cómo ilustrar las enseñanzas más técnicas de la Economía, como pueden ser la Estadística o la Econometría, a partir del uso de noticias de prensa de tal modo que los alumnos perciban que esas técnicas no son simplemente parte del mundo de la abstracción sino que, de hecho, son herramientas fundamentales que tienen una clara utilidad para hacer frente y obtener análisis rigurosos de los problemas económicos del mundo real.

\section{Referencias bibliográficas}

ASOCIACIÓN AMERICANA DE ESTADÍSTICA (2010): Guidelines for assessment and instruction in Statistics education. College report: http://www.amstat.org/education/gaise/ [fecha de consulta: 27 de diciembre de 2011].

BECKER, William E. (1997): "Teaching Economics to undergraduates". Journal of Economic Literature, no 35 , pp. 1347-1373.

BECKER, William E. (2002a): "Engaging students in quantitative analysis with short case examples from the academic and popular press". American Economic Review, $\mathrm{n}^{\mathrm{o}} 8(2), \mathrm{pp} .480-486$.

BECKER, William E. (2002b): "Teaching quantitative methods in Economics: Alternatives to theorem and proof and chalk and talk". Economic Analysis and Policy, $\mathrm{n}^{\circ}$ 32(2), pp. 159-180.

BECKER, William E. y GREENE, William H. (2001): "Teaching Statistics and Econometrics to undergraduates". Journal of Economic Perspectives, ${ }^{\circ}$ 15(4), pp. 169182.

BECKER, William E. y WATTS, Michael (2001): "Teaching Economics at the start of the $21^{\text {st }}$ century: Still chalk-and-talk". American Economic Review, $\mathrm{n}^{\circ}$ 91(2), pp. 446-451.

CUADRADO ROURA, Juan Ramón (2010): “Tres preguntas sobre la Economía como ciencia y como práctica". Documentos de Trabajo IAES, nº 09/2010.

DOW, Sheila C.; EARL, Peter E.; FOSTER, John; HARCOURT, Geoffrey C.; HODGSON, Geoffrey M.; METCALFE, J. Stanley; ORMEROD, Paul; ROSEWELL, Briget; SAWYER, Malcom C.; y TYLECOTE, Andrew (2009): Carta a la Reina de Inglaterra de un grupo de economistas británicos: http://empresa.euroresidentes.com/2009/07/los-economistas-y-la-prevision-de-la.html [fecha de consulta: 27 de diciembre de 2011].

EL PAÍS (2011): "El aumento de la desigualdad en España", en El País. 30 de octubre: http://www.elpais.com/graficos/economia/aumento/desigualdad/Espana/elpepueco/20111030elpepueco_1/Ges/ [fecha de consulta: 4 de noviembre de 2012].

FRIEDMAN, Milton (1999): “Conversation with Milton Friedman”, en SNOWDON, Brian y VANE, Howard R.: Conversations with leading economists: Interpreting modern macroeconomics. Cheltenham, Edward Elgar. 
FUNDACIÓN BBVA (2010): Estudio internacional Fundación BBVA sobre estudiantes universitarios de seis países europeos. Madrid, Fundación BBVA.

FUNDACIÓN FOESSA (2012): Exclusión y desarrollo social en España. Análisis y perspectivas 2012. Madrid, Cáritas.

INSTITUTO NACIONAL DE ESTADÍSTICA (2012): Encuesta de condiciones de vida, nota de prensa sobre los resultados provisionales: http://www.ine.es /prensa/np740.pdf [fecha de consulta: 4 de noviembre de 2012].

KRUGMAN, Paul (2012): "Si Alemania no cede, el euro se rompe", en La Vanguardia, 8 de julio: http://www.lavanguardia.com/economia/20120708/54322010105 /paul-krugman-si-alemania-no-cede-el-euro-se-rompe.html [fecha de consulta: 10 de julio de 2012].

MARTÍNEZ FERNÁNDEZ, Valentín Alejandro; JUANATEY BOGA, Óscar; y COSTA SÁNCHEZ, Carmen (2012): "Agenda setting y crisis económica: Influencia de la prensa en el comportamiento de consumo y ahorro". Estudios sobre el mensaje periodístico, Vol. 18, Núm. 1, págs.: 147-156. Madrid, Servicio de Publicaciones de la Universidad Complutense. <http://dx.doi.org/10.5209 /rev_ESMP.2012.v18.n1.39362>

ORGANIZACIÓN DE CONSUMIDORES Y USUARIOS (2011): 10 años del euro: Precios que suben y no bajan: http://www.ocu.org/10-anos-del-euro-precios-quesuben-y-no-bajan-s565514.htm [fecha de consulta: 30 de diciembre de 2011].

WATTS, Michael y SCHAUR, Georg (2011): "Teaching and assessment methods in undergraduate Economics: A fourth national quinquennial survey". Journal of Economic Education, nº 42(3), pp. 294-309.

\section{María Rosalía VICENTE CUERVO}

Universidad de Oviedo

Departamento de Economía Aplicada

Profesora Titular de Universidad

mrosalia@uniovi.es 